\title{
SOZIALSTAAT UND SOZIALPOLITIK IN DER TURKEI
}

\author{
Prof. Dr. Sait DlLIK
}

\section{Der Begriff}

Zur besseren Verstăndigung des Themas soll zunächst mit einigen Worten der Begriff Sozialstaat ${ }^{1}$ geklärt bzw. bestimmt werden:

Auf die Spuren des Begriffs "Sozialstaat" stoßen wir in der franzobsischen Verfassung von 1946 und der italienischen Verfassung von 1947.

In der Präambel der franzősischen Verfassung von 1946 wird erklăr, dass die Nation die notwendigen Voraussetzungen zur Entwicklung des Menschen und der Familie schafft. Mit dieser Verfassung wird ausserdem die Gründung von sozialen, beruflichen, wirtschaftlichen und örllichen Errichtungen (Vereine, Verbände) erlaubt.

In der italienischen Verfassung von 1947 wird bestimmt, daB der Staat alle wirtschaftlichen und sozialen Hindernisse abzuschaffen hat, die den Bürger beschränkend und Gleichheiten zerstörend die Entwicklung des Menschen hindern.

Allerdings wird in diesen Verfassungen der Ausdruck "Sozialstaat" als solcher noch nicht gebraucht.

Der Ausdruck wird zum ersten Mal in der Welt im Deutschen Grundgesetz vom 1949 offen verwendet und gewinnt damit aktuelle Bedeutung. Artikel 20 I des deutschen Grundgesetzes bezeichnet die Bundesrepublik Deutschland als den sozialen Staat. Ausserdem besagt der Artikel 28, daß die verfassungsmäßige Ordnung der deutschen Lảnder der eines "sozialen" Rechtsstaates entsprechen muss.

\footnotetext{
* Yuksekőğretim Denetleme Kurulu Úyesi

${ }^{1}$ Zu Erklärungen über den Begriff Sozialstaat siehe $H$. Peters, Sozialstaat, in: Staatslexikon, siebter Band, Freiburg i. Br. 1962, s. 394-395; E. Forsthoff (hg), Rechtsstaatlichkeit und Sozialstaatlichkeit, 1968, A. Göze, Liberal, Marxist, Faşist ve Sosyal Devlet Sistemleri (Die liberalen, marxistischen, faschistischen und sozialen Staatssysteme), Istanbul 1977, S. 155 ff.
} 
Der Terminus "Sozialstaat" wird auch in den türkischen Verfassungsgesetzen von 1961 und 1982 verwendet.

Fast alle modernen Staaten nehmen auch, obwohl in ihren Verfassungsgesetzen dieser Ausdruck offen nicht gebraucht wird, Sozialstaatlichkeit mit Recht für sich in Anspruch.

Der Inhalt und Umfang bzw. die Bedeutug des Sozialstaates sind im allgemeinen wie auch in Deutschland viel umstritten. Nach manchen Autoren besitzt der Begriff Sozialstaat keinerlei juristische Bedeutung. Nach anderen bildet dieser Begriff nur eine an das Ermessen des Staates gerichtete und für Gesetzesauslegung verbindliche Zielbestimmung. Wieder andere Autoren verstehen unter Sozialstaat lediglich einen politisch-programmatischen und rechtlich unverbindlichen Begriff. Moderne sozialgesinnte Autoren erkennen dagegen im Sozialstaat einen wichtigen verfassungsrechtlichen Begriff. Nach diesen bildet Sozialstaat ein ethisch und politisch fundiertes Verfassungsprinzip. Damit wird die Realisierung und Beachtung des Sozialstaates den gesetzgebenden-, rechtsprechenden- und Verwaltungsorganen des Staates zur Pflicht gemacht. Diese Auslegung findet mit Recht eine allgemeine Anerkennung. Dadurch wird der Übertreibung anderer Grundprinzipien der Verfasung, wie z.B. freie Entfaltung der Persönlichkeit, Gleichheit, Rechtsstaat rechtlich wirksam entgegengewirkt.

Man nennt einen Staat sozial, wenn er im entscheidender Maßstab Bestrebung hat, die materielle und idcelle Not zu beseitigen. Diese Bestrebung richtet sich nicht nur auf die Hilfe für wirtschaftlich schwachen Bevőlkerungsteil. Auch die wirtschaftlich nicht schwache Bevőlkerung könnte z.B. im Alter oder sonst irgendwann mal in materielle Not fallen. Deshalb brauchen neben Bedürftigen auch die Nichtbedürfige Sicherheit gegen soziale Gefahren des Lebens. Deshalb ist die Gewährleistung der sozialen Sicherheit gegen Risiken wie Krankheiten, Unfälle, Mutterschaft, Arbeitslosigkeit, Alter, Invalidităt und Tod sowie Kinderreichheit für jedermann Aufgabe des Sozialstaates.

Auch weitere Maßnahmen wie Erwachsenenbildung, Verbesserung der Bildungschancen, Verbreitung der höheren- und Hochschulbildung, Schutz der Arbeit und Arbeitenden, hygienische, sportliche und jugendfordernde Vorkehrungen, Sicherung des Rechts auf Arbeit und freie. Arbeitswahl, Gewährleistung der Lohngerechtigkeit, Umverteilung des Einkommens, Schaffung gesunder Wohnverhältnisse, Anerkennung bzw. Sicherung des Rechtes auf Gründung von Gewerkschaften und Arbeitgeberverbänden, Schließung von Tarifverträgen, Ausübung von Streik (und Aussperrung) und Mitbestimmung gehören zu dem pflichtbereich des Sozialstaates.

In den Entwicklungsländern ist dazu noch die Aufgabe des Sozialstaates, neben obigen sozialen Maßnahmen wirtschaftliche Entwicklung des Landes voranzutreiben, damit zur Verwirklichung sozialer Zwecken mehr Quellen zur Verfügung stehen.

Die Aufgaben des Sozialstaates bilden, wie auch bei anderen Grundprinzipien der Verfassung der Fall ist, als solche keine klagbaren subjektiven Rechte für Einzelne. So kann z.B. die Beeinträchtigung des Sozialstaats-Prinzips nur mit zur Begründung eines sonstwie verletzten Anspruchs herangezogen werden. Sie führt aber prinzipiell nicht zur Verfassungswidrigkeit von Gesetzen oder einzelnen Maßnahmen. Die rechtliche Wirksamkeit des Sozialstaat-Grundsatzes wird jedoch kraft unmittelbar geltenden 
objektiven und positiven Rechts sichergestellt. Dieses Recht bürdet dem Staat auf, nicht passiv zu bleiben, sondem zur Realisierung der sozialen Grundsătze aktiv tătig zu werden.

Somit ist für den Gesetzgeber bindend gemacht, notwendige Gesetze zur Verwirklichung des Sozialstaates zu erlassen. Auch die Verwaltungsbehörden haben im Rahmen ihrer freien Gestaltungsmoglichkeiten als auch bei Ausführung der Gesetze die sozialen Aufgaben bzw. die soziale Zielsetzung, die die Verfassung dem Staat auferlegt, zu beachten sowie zu erfüllen. Schließlich sind die Gerichte bei der Rechtsanwendung, wo der Gesetzeswortlaut mehrere Enstscheidungen zuläßt, verpflichtet soziale Gedanken und Prinzipien zu berücksichtigen.

Kein Verfassungsgrundsatz kann alleine für sich gelten. Die sozialstaatlichen Grundsătze begrenzen, wie schon erklärt, andere Grundrechte der Verfassung. Sie werden jedoch wiederum auch von diesen beschränkt. Damit wird ein Gleichgewicht zwischen sozialen und anderen Grundrechten hergestellt.

Der Sozialstaat bekennt sich zu demokratisch politischen Grundsătzen sowie zur marktwirtschaftlichen Ordnung. Er bezweckt lediglich, die sozialen Mängel der klassichen Demokratie sowie die der Marktwirtschaft zu beheben.

Nach diesen Erklärungen über den Begriff des Sozialstaates würde ich mir erlauben, den anderen Bestandteil meines Themas, den Begriff der Sozialpolitik, als angewande Sozialpolitik zu definieren, als Gesamtheit der Maßnahmen bzw. der Politik zur Verwirklichung des Sozialstaates. Türkei.

Nun kommen wir zum eigentlichen Thema Sozialstaat und Sozialpolitik in der

In der Präambel des türkischen Verfassungsgesetzes von 1982 lauten die Absătze 6 und 7 wie folgt:

"Jeder türkische Staatsbürger genießt gemaß den Erfordernissen der Gleichheit und der sozialen Gerechtigkeit die Grundrechte und Grundfreiheiten der tïrkischen Verfassung. Er hat von seiner Geburt an das Recht und die Möglichkeit, innerhalb der nationalen Kultur-, Zivilisations- und Rechtsordnung ein würdiges Leben zu führen und seine materielle und geistige Existenz in diesem Sinne zu entfalten.

Die türkischen Staasbürger sind insgesamt in nationalem Stolz, in nationaler Freude und nationalem Leid, in ihren Rechten und Pflichten gegenüber dem nationalen Dasein in Segen und Mühsal sowie in jeglicher Manifestation des Nationallebens geeint. Sie haben das Recht in den Gefühlen der absoluten Achtung des Rechts und der Freiheit des anderen und der gegenseitigen herzlichen Liebe und Brüderlichkeit sowie im Verlangen und Glauben an "Frieden im Lande- Frieden in der Welt" ein heilvolles Leben führeń zu wollen."

Der Artikel 2 des tïrkischen Verfassungsgesetzes lautet:

"Die türkische Republik ist ein im Geiste des Friedens der Gemeinschaft, der nationalen Solidarităt und der Gerechtigkeit, die Menschenrechte achtender, dem 
Nationalismus Atatürks verbundener und auf den in der Prăambel verkündeten Grundprinzipien beruhender demokratischer, laizisticher und sozialer Rechtstaat."

Aus oben angegebener Präambel und Artikel 2 der türkischen Verfassung geht hervor, daß die Türkische Republik als ein Sozialstaat bezeichnet wurde und die türkischen Staatsbürger die Grundrechte und - Freiheiten nach dem Maß sozialer Gerechtigkeit genießen sollen. Aus dem Obigen geht hervor, daß die Sozialstaatlichkeit der türkischen Republik in ihrer Verfassung verankert ist.

\section{Was versteht man unter Sozialstaat in der Türkei?}

Um dies festzustellen brauchen wir, keine wissenschaftlichen Diskussionen und Auseinandersetzungen. Der Begriff Sozialstaat ist nähmlich in der türkischen Verfassung durch verschiedene Artikel ausreichend bestimmt.

In den Artikeln 41-65 des Verfassungsesetzes werden die sozialen und wirtschaftlichen Rechte und Aufgaben geregelt. Der eigentliche Inhalt und Umfang der Sozialstaatlichkeit der türkischen Verfassung sind hier bestimmt.

Weitere Artikel, wie Artikel 35 über Eigentumsrecht, Artikel 171 über Fortentwicklung des Genossenschaftswesens und Artikel 172 sowie 173 über Schutz der Verbraucher, Kleinhändler und Handwerker ergänzen den Inhalt und Umfang der sozialen Staatlichkeit der türkischen Verfassung. Ähnliche Bestimmungen wie die der heutigen Verfassung hatte auch das Verfassungsgesetz von 1961. Die Sozialstaatlichkeit in diesem Sinne besteht also in der Türkei schon seit 1961.

Der Inhalt und Umfang der Sozialstaatlichkeit der türkischen Verfassung, die die oben erwahnten Artikel bestimmen, decken sich mit einer Ausnahme gut mit wisssenschaftlichen Folgerungen und Deutungen modemer Autoren über den Inhalt und Umfang des Begriffs Sozialstaat. Diese Ausnahme bildet die Mitbestimmung. Darüber hat die tïrkische Verfassung keine Bestimmung gebracht.

Jetzt wollen wir den Inhalt und Umfang der Sozialstaatlichkeit und die Sozialpolitik in der Türkei unter Handhabung der oben erwăhnten Bestimmungen der türkischen Verfassung und Gruppierung nach sozialpolitischen Fachthemen einzeln untersuchen.

\section{Schutz der Familie}

Nach türkischer Verfassung bildet die Familie den Grund der türkischen Gesellschaft.

Der Staat trifft die notwendigen MaBnahmen und gründet die notwendigen Einrichtungen, um das Wohl und Heil der Familie sowie insbesondere den Schutz der Mutter und Kinder und die Lehre und Anwendung der Familienplanung zu gewăhrleisten." 2

\footnotetext{
2 Verfassungsgesetz, Art. 41.
} 
Im Arbeitsgesetz sowie in den demgemäß erlassenen Verordnungen gibt es Bestimmungen zum Schutze der Mütter, Kinder und Frauen. Durch diese Bestimmungen werden Kinder, Mütter und die Frauen besonders geschützt. Es gibt auch staatliche Zentren für Gesundheit von werdenden Müttern und Kindern. Dort werden die werdenden Mütter kostenlos untersucht, Kinder geimpft.

Für die Familienplanung wird viel getan. Seitens der zustăndigen Behörden, die im Land weit verbreitet sind, werden Verhütungsmittel für Empfängnis verteilt und die Bevolkerung aufgeklärt. Es gibt zur Zeit ausserdem über 70 Ausbildungszentren für Familienplanung. Auch manche privaten Vereine zeigen Bestrebungen nach Verbreitung der Familienplanung. Im Osten und Südosten sind aber die Effizienz der Anstrengungen für Familienplanung sehr gering.

\section{Bildung und Erziehung}

Nach türkischer Verfassung darf "niemandem sein Recht auf Erziehung und Bildung verweigert werden.

Der Umfang des Rechts auf Bildung wird durch Gesetz bestimmt und geregelL.

Die Grundschulausbildung ist für alle weiblichen und männlichen Staatsbürger Pflicht und in den staatlichen Schulen kostenlos.

Der Staat läßt den mittellosen erfolgreichen Schülern, um die Fortsetzung ihrer Ausbildung zu ermogglichen, durch Stipendien oder auf anderen Wegen die notwendige Unterstützung zuteil werden. Der Staat trifft die Maßnahmen, um diejenigen, deren Zustand eine Sondererziehung erfordert, für die Gemeinschaft nützlich werden zu lassen."

Alle staatlichen Grund- und hörere Schulen sind in der Türkei, entsprechend den oben angegebenen Bestimmungen der Verfassung, kostenlos. Neben den Staatlichen bestehen auch Privatschulen, die gegen Entgelt sind. Die Zahl der Privatschulen ist allerdings im Verhälınis zu staatlichen Schulen sehr gering.

Die staatlichen Grund- und höhere Schulen erleiden seit den 70 er Jahren stets Qualitătseinbusse. Der Grund dafür liegt in der schnellen Vermehrung der Bevőlkerung, schlechten Besoldung der Lehrer sowie im mit diesen Faktoren verbundenen Mangel an Lehrern, besonders an qualifizierten Lehrkräften.

Es gibt auch Sonderschulen für behinderte, deren Zahl jedoch davon entfernt ist, den Bedarf zu decken.

Die Hochschulbildung ist seit den 80 er Jahren nich mehr kostenlos. Die Studiengebühren bilden allerdings einen kleinen Bruchteil der tatsăchlichen Kosten des jeweiligen Studienplatzes. Außerdem wird fast allen Bewerbern Kredit für Studiengebühren gewährt. Andererseits gibt es Stipendien und Kredite zur Finanzierung des Studiums. Die Kredite werden großzügigerweise einer breiten Schicht gewăhrt, wenn

${ }^{3}$ Verfassungsgesetz, Art. 42. 
auch ihre Höhe infolge der Finanzschwierigkeiten des Staates und Inflation oft nicht ausreichend ist.

In der Türkei gibt es auch private Universităten, die seitens der Stiftungen, die keinen Gewinn bezwecken dürfen, gegründet sind. Diese Universitäten sind entgeltlich. Auch diese Universităten gewähren qualifizierten Studenten Stipendien.

Die Schulbesuchsquoten betragen 1995 für Grundschulen 100, Mittelschulen 65,5, allegemeine Gymnasien 30,6, beruflich - technische Gymnasien 22,4 und für Hochschulen 12,6\% (einschliesslich des Fernstudiums $22,1 \%$ ). ${ }^{4}$

\section{Gebrauch des Eigentumsrechts}

Nach türkischer Verfassung "genießt jedermann das Recht auf Eigentum und Erbe.

Diese Rechte können nur im offentlichen Interesse durch Gesetz beschrănkt. werden.

Der Gebrauch des Rechts auf Eigentum darf außerdem dem Gemeinwohl nicht entgegenstehen."5

Damit wird m.E. besagt, daß der Gebrauch von Eigentum mit dem Grundsatz Sozialstaatlichkeit eingeschränkt werden muss. Dementsprechend haben der Gesetzgeber, die Gerichte und Verwaltungsbehörden in ihrer Tätigkeit darauf zu achten.

Bodeneigentum und Schutz der Unabhängig Arbeitenden in der Landwirtschaft

Die türkische Verfassung hat Bestimmungen über Schutz, fruchtbringende Bewirtschaftung und -Verteilung des Bodens sowie über Förderung der Landwirte.

Hinsichtlich des Bodeneigentums sieht das Verfassungsgesetz folgendes vor: "Der Staat trifft die notwendigen Maßnahmen, um die fruchtbringende Bewirtschaftung des Bodens zu schützen und zu entwickeln, seinen Verlust durch Erosion zu verhindern und dem Bauem, der einen Hof ohne oder mit nicht ausreichendem Boden betreibt, Boden zu verschaffen. Das Gesetz kann zu diesem Zweck den Umfang des Bodens entsprechend den verschiedenen Landwirtschaftszonen und -arten bestimmen. Die Beschaffung von Boden für den Bauern ohne oder mit unausreichendem Boden darf nicht zu einem Rückgang der Produktion, der Verkleinerung der Wälder und der Verringerung der übrigen Böden und Bodenschätze führen.

Die zu diesem Zweck verteilten Böden dürfen nicht geteilt und nicht außerhalb der erbrechtlichen Vorschriften an andere übertragen sowie nur von den Bauern, an welche die Verteilung erfolgt ist, und ihren Erben bewirtschaftet werden. Die Grundsätze hinsichtlich

\footnotetext{
${ }^{4}$ Die Zahlen sind entnommen aus dem Jahresprogramm 1996 vom Staatlichen Planungsamt, Resmi Gazete (Staats-Anzeiger) von 2 Juni 1996, S. 22.

${ }^{5}$ Verfassungsgesetz, Art. 35.
} 
der Rücknahme des verteilten Bodens durch den Staat für den Fall des Wegfalls dieser Bedingungen werden durch Gesetz geregelt." 6

Im Sinne dieser Bestimmungen der Verfassung hat es schon manche Bestrebungen gegeben und konnte einiges durchgeführt bzw. einige Böden verteilt werden.

Schon im Jahre 1945 wurde ein Gesetz zur Bodenbeschaffung für Bauem erlassen. Zwischen 1947-1962 wurde 377354 Bauemfamilien 18.067.666 Dekar Boden verteilt.

Nach 1960 gewann die Bodenverteilung wieder Aktualităt. Die Verfassung von 1961 brachte schon damals über Bodeneigentum ăhnliche Bestimmungen wie die der Verfassung von 1982. Aber nach langem Zogern konnte erst 1973 das Gesetz für Bodenund Landwirtschaftsreform erlassen werden. Das Gesetz bezweckte zugleich Bodenverteilung und Verhinderung der Flurbildung. Die Bestimmungen des Gesetzes wurden versuchsweise hauptsächlich im Gebiet der südőstlichen Stadt Urfa sowie in manchen anderen Stadtgebieten durchgeführt. Dicses Gesetz wurde aber 1976 vom Verfassungsgericht formwegen ausser Kraft gesetzt.

Auf Grund dieses Gesetzes konnte zwar genügend Boden von Großgrundbesitzern zum $Z$ wecke der Verteilung verstaatlicht werden. Die Verteilung dieses Bodens unter den Bauem ohne ausreichenden Boden konnte aber wegen der Abschaffung des Gesetzes nicht zu Ende geführt werden. Der Kleinbauernfamilien verteilte Boden bildete nur einen kleinen Bruchteil von $6,1 \%$ des verstaatlichten Bodens (in Urfa 1175 Bauernfamilien 176548, in Ankara 74 Bauemfamilien 5820 Dekar Boden). Der übrige Teil von 93,9 \% blieb in Händen des Staates. ${ }^{7}$

Es wurde dann 1984 cin Gesetz über Bodenregelung in den Bewässerungsgebieten und Landwirtschaftsreform erlassen. Auch in diesem Gesetz wurde Bodenverteilung für Bauemfamilien ohne genügenden Boden vorgesehen.

Die türkische Verfassung bringt auch Bestimmungen über Ackerbau, Viehzucht und Schutz für die Arbeitende in diesem Sektor. Sie lauten:

"Mit dem Ziel, den zweckentfremdeten Gebrauch und die Zerstörung der Äcker, Wiesen und Weiden zu verhindern und gemăß den Prinzipien der landwirtschaftlichen Produktionsplanung die Pflanzen- und Viehproduktion zu erhöhen, erleichtert der Staat den Betriebern von Landwirtschaft und Viehzucht die Beschaffung von Geräten und Materialien zur Bewirtschaftung und von anderen Mitteln.

Der Staat trifft die Maßnahmen, welche notwendig sind, damit die pflanzlichen und tierischen Produkte bewertet werden und ihr wahrer Wert in die Hände der Produzenten gelangt." 8

\footnotetext{
${ }^{6}$ Verfassungsgesetz, Art. 44.

${ }^{7}$ Zur Einzelheiten Uber Bodenreform und -Verteilung siehe N. Kuyucuklu, Turkiye Ekonomisi (Die türkische Wirtschaft), Istanbul 1986, S. $305 \mathrm{ff}$.

8 Verfassungsgesetz, Art. 45.
} 
Zur Verwirklichung dieser Ziele strebt man schon seit ersten Jahren der Republik und besonders seit den 50 er Jahren. Seit 30 er Jahren wird seitens der staatlichen Landwirtschaftsbank direkt oder durch landwirtschaftliche Kreditgenossenschaften und spăter auch seitens der Anstalt für Landwirtschafuliche Ausstattung den Landwirten zur Beschaffung von Gerăten, Materialien und Viehbeständen sowie zur Erhöhung der Produktivităt in der Landwirtschaft, Viehzucht und neuerdings in Fischerei Kredite gewăhrt.

Andererseits werden mit Krediten seitens der landwirtschaftlichen Vertriebsgenossenschaften, mit staatlich festgelegten Mindestpreisen, durch den Ankauf der Erzeugnisse seitens des Amtes für Landwirtschaftliche Erzeugnisse und der anderen staatlichen Stellen sowie seitens der Vertriebsgenossenschaften Landwirte gefordert.

\section{Schutz der unabhängig Arbeitenden im Handel und Handwerk}

Nach der Verfassung "hat der Staat die Maßnahmen zum Schutz und zur Unterstützung der Kleinhändler und Hadwerker zu treffen."9

Eine zu diesem Zweck errichtete Bank, die Volksbank, gewährt rund drei Millionen Kleinhändlern und Handwerkem verschiedene Kredite mit sehr niedrigen Zinsen, die oft weit unter den Inflationsraten stehen. Außerdem gibt es verschiedene Projekte zur Entwicklung und Entfaltung der Kleinhändler und Handwerker. Eine staatliche Organisation zur Entwicklung und Förderung von kleinen und mittelständischen Betrieben leistet dieser Gruppe finanzielle und technische Dienste sowie berăt sie in der Geschäftsführung. ${ }^{10}$

\section{Schutz der Verbraucher}

Die türkische Verfassung sieht auch zur Förderung der Verbraucher Maßnahmen vor:

Danach "hat der Staat Maßnahmen zum Schutze und zur Aufklärung der Verbraucher zu treffen. Er fördert die Selbstschutzaktivitäten der Verbraucher." 11

In dieser Beziehung hat man in der Türkei bis 1995 praktisch keine Maßnahmen getroffen. Seit den 90 er Jahren gewann aber dieses Thema an Aktualitat und wurde viel diskutiert. Schließlich wurde im Februar 1995 das Gesetz zum Schutze der Verbraucher erlassen. Beim Einbringen dieses Gesetzes spielte auch der bevorstehende Eintritt in die Zollunion mit EU-Ländern eine Rolle.

\footnotetext{
${ }^{9}$ Verfassungsgesetz, Art. 173.

${ }^{10}$ Dazu siehe Tes - Ar und Türkiye Halk Bankası, Türkiye Halk Bankası Tarafından Küçük ve Orta Olçekli Işletmeler ile Esnaf ve Sanatkarlara Sunulan Krediler ve DanışmanlıkEł̆gitim Hizmetleri (Die Kredite und Beratungs-und Ausbildungsdienste, die von der Türkischen Volksbank den kleinen und mittelständischen Betrieben sowie Kleinhändlern und Handwerkern gewährt bzw. geleistet werden), Ankara 1994.
}

11 Verfassungsgesetz, Art. 172. 
Dieses Gesetz konnte aber bis heute nicht in vollem Unfang ausgefuhrt werden. Der Grund liegt nicht zuletzt daran, daß sich auch die Verbraucher noch nicht organisiert haben.

Eine weitere Bestimmung der tilrkischen Verfassung sieht ebenfalls MaBnahmen zum Schutze der Verbraucher vor. Danach "trifft der Staat unter Beachtung der Interessen der nationalen Wirtschaft die Maßnahmen zur Fortentwicklung der Genossenschaften mit dem Zweck, vorranging die Produktion zu erhohen und den Verbraucher zu schützen. ${ }^{12}$

Der Staat fördert dementsprechend mit verschiedenen Maßnahmen die Verbrauchergenossenschaften sowie die Selbstschutzaktivităten der Verbraucher.

\section{Schutz der abhängig Arbeitenden}

Die türkische Verfassung sieht in dieser Hinsicht als erstes die Freiheit der Arbeit und des Vertragsschlusses vor. Danach "genießt Jedermann die Freiheit, in einem belibiegen Bereich Arbeit aufzunehmen und Verträge zu schließen. Die Gründung von Privatunternchmen ist frei.

Der Staat trifft Maßnahmen zur Gewăhrleistung einer den Erfordemissen der nationalen Wirtschaft und den sozialen Zielen entsprechenden Funktionierung und Arbeit der Privatunternehmen in Sicherheit und Stabilität." 13

Dieser Grundsatz der Verfassung wird durch marktwirtschaftliche Ordnung der türkischen Wirtschaft verwirklicht.

Nach türkischer Verfassung "ist die Arbeit Jedermanns Recht und Pflicht. Der Staat trifft zur Erhöhung des Lebensstandards der Arbeitenden, sowie zur Entfaltung des Arbeitslebens die notwendigen Maßnahmen, um ein wirtschätliches Ambiente zu schaffen, das den Schutz der Arbeitenden, die Förderung der Asbeit und die Verhinderung der Arbeitslosigkeit begünstigt.

Der Staat trifft die Maßnahmen zur Erleichterung und zum Schutz des Arbeitsfriedens in den Beziehungen zwischen Arbeitnehmem und Arbeitgebern. ${ }^{14}$

Die Verwirklichung dieses Grundsatzes der türkischen Verfassung sollte hauptsächlich durch eine zweckvolle Entwicklungs-, Konjunktur-, Beschäftigungs-und allgemeine Wirtschaftspolitik sowie durch eine Umverteilungspolitik im Rahmen der marktwirtschaftlichen Ordnung erwirkt werden. Man kann aber sagen, daß die tïrkischen Regierungen diese Politiken nicht immer erfolgreich durchführen konnten. Trotzdem hat die türkische. Wirtschaft stăndig Fortschritte gemacht und demgemäß konnte der Lebensstandard des Volkes bzw. der Arbeiter erhöht werden, wenn dies auch nicht immer im gewünschten und möglichen Maße erfolgte.

12 Verfassungsgesetz, Art. 171.

13 Verfassungsgesetz, Art. 48.

${ }^{14}$ Verfassungsgesetz, Art. 49. 
Nach türkischer Verfassung "sichert der Staat gesunde Arbeitsbedingungen und Recht auf Erholung. Niemand darf mit Arbeiten beschäftigt werden, die mit seinem Alter, seinem Geschlecht und seiner Kraft nicht vereinbar sind.

Minderjăhrige und Frauen sowie körperlich und geistig Behinderte werden im Hinblick auf die Arbeitsbedingungen besonders geschützt.

Erholung ist das Recht der Arbeitenden.

Das Recht auf bezahiten Wochenend- und Feiertagsurlaub sowie auf bezahlten Jahresurlaub und die Bedingungen hierzu werden durch Gesetz geregelt."15

Um die Verwirklichung dieser Grundsătze hat man sich schon seit ersten Jahren der Republik bemüht. Zu diesem Zweck brachte man unter anderem 1936 Arbeitsgesetz, 1946 Gesetz zur Gründung des Arbeitsamtes, 1946 Gesetz über Gründung des Arbeitsministeriums, 1950 Gesetz zur Gründung von Arbeitsgerichten, 1952 Arbeitsgesetz für Arbeitnehmer und Arbeitgeber in der Presse, 1954 Arbeitsgesetz für Seeleute, 1977 Gesetz für Lehrlinge, Gesellen und Meister ein.

Diese Gesetze wurden mit der Zeit geăndert, ergănzt oder durch neue Gesetze ersetzt. Auf diese Weise wurden die Bestimmungen der Gesetze der Zeit, bzw. den Verfassungen angepaßt.

Das heute gültige Arbeitsgesetz ${ }^{16}$ sieht hinsichtlich des Schutzes der abhängig Arbeitenden viele Maßnahmen vor. Diese Schutzmaßnahmen umfassen vor allem die Kinderarbeit, die Frauenarbeit, die Arbeit in der Mutterschaft, die Arbeit von Jugendlichen, Behinderten und Vorbestraften, die Regelung und Kürzung der Arbeitszeit, den Wochenend-, Feiertags- und bezahlten Jahressurlaub, sowie den technischen Arbeitsschutz, d.h. Arbeitshygiene und Gefahrenschutz im Betrieb.

Außerdem gibt es kostenlose Arbeitsvermittlungsdienste durch Arbeitsămter sowie Arbeitsgerichte zum besseren Schutz der Rechte der Arbeitenden. Die Effizienz der Arbeitssämter ist allerdings in der Türkei nicht groß.

\section{Berufsverbände und kollektive Arbeitsbeziehungen}

Die türkische Verfassung erkennt das Recht auf Berufsverbănde und kollektive Arbeitsbeziehungen. Sie bringt Bestimmungen über Gewerkschaften, Arbeitgeberverbănde und ihre Dachverbände sowie Bestimmungen über Tarifverträge, Streik und Aussperrung. Diese Bestimmungen lauten wie folgt:

"Die Arbeitnehmer und Arbeitgeber haben das Recht, ohne vorherige Erlaubnis Gewerkschaften, Arbeitgeberverbände sowie Dachverbände zu gründen, um die wirtschaftlichen und sozialen Rechte und Interessen innerhalb der Arbeitsbeziehungen ihrer Mitglieder zu schülzen und zu entfalten.

\footnotetext{
15 Verfassungsgesetz, Art. 50.

$16_{1475}$ Sayılı Is Kanunu (Arbeitsgesetz Nr. 1475) vom 25.8.1971.
} 
Um die Gewerkschaften, Arbeitgeberverbände und Dachverbände zu gründen, genügt es, die im Gesetz bestimmten Informationen und Dokumente bei der im Gesetz aufgeführten zustăndigen Behörde einzureichen. Im Falle der Feststellung der Gesetzwidrigkeit dieser Informationen und Dokumente ruft die zusländige Behorde wegen der Aussetzug der Betătigung oder der Schließung der Gewerkschaften bzw. der Verbände das Gericht an.

Die Eintritu in die Gewerkschaften und die Verbănde sowie der Austritt sind frei.

Niemand darf gezwungen werden in einem Berufsverband Mitglied zu werden, zu bleiben oder daraus auszutreten.

Arbeitnehmer und Arbeitgeber dürfen zur gleichen Zeit in nicht mehr als einer Gewerkschaft bzw. einem Arbeitgeberverband Mitglied sein.

Um an irgendeinem Arbeitsplatz beschäftigt zu werden, darf als Voraussetzung nicht die Mitgliedschaft oder Nichtmitgliedschaft in einer Gewerkschaft verlangt werden.

Um eine führende Funktion in Gewerkschaften oder ihren Dachverbänden übernehmen zu können, ist es notwendig, effektiv zehn Jahre als Arbeitnehmer gearbeitet zu haben.

Satzungen, Verwaltung und Geschäfte der Gewerkschaften, Arbeitgeberverbände und ihrer Dachverbănde dürfen den Prinzipien der Republik und den demokratischen Grundsătzen, die in der Verfassung bestimmt sind, nicht entgegenstehen." 17

"Arbeitnehmer und Arbeitgeber haben das Recht, zur gegenseitigen Regelung ihrer wirtschafulichen und sozialen Lage und Arbeitsbedingungen Tarifverträge abzuschließen.

Wie der Tarifvertag abzuschließen ist, wird durch Gesetz geregelt.

Auf einem Arbeitsplatz darf für dieselbe Periode nicht mehr als ein Tarifvertrag abgeschlossen und angewendet werden." 18

Nach einer Verănderung der türkischen Verfassung im jahre 1995 dürfen die Beamten Gewerkschaften und Dachverbănde gründen, im Namen ihrer Mitglieder Gerichte anrufen, mit den Verwaltungsbehorden entsprechend ihren Zielen Gesamtarbeitsverhandlungen führen. Nach diesen Verhandlungen wird über die Übereinstimmung oder Nichtübereinstimmung ein Protokoll unterschrieben. In diesem Protokoll werden die Konfliktpunkte gezeigt. Dieses Protokoll wird dem Ministerrat vorgelegt. Der Ministerrat entscheidet den Fall, unabhängig von diesem Protokoll und unternimmt notwendiges.

\footnotetext{
17 Verfassungsgesetz, Art. 51.

18 Verfassungsgesetz, Art. 53 .
} 
Wie aus obiger Erklärung zu verstehen ist, können die Beamten in der Türkei zwar Gewerkschaften und Dachverbände gründen; sie dürfen aber keinen Tarifvertrag schließen und keinen Streik ausüben. 19

"Bei Auftreten eines Konfliktes wăhrend des Abschlusses eines Tarifvertrages haben die Arbeitnehmer das Streikrecht. Verfahren, Bedingungen, Umgang und Ausnahmen des Gebrauchs des Streikrechts und der Anwendung der Aussperrung durch den Arbeitgeber werden durch Gesetz geregelt.

Das Streikrecht und die Aussperrung dürfen nicht in einer gegen die Regeln von Treue und Glauben verstossenden Weise, zum Schaden der Gemeinschaft und in einer das nationale Vermőgen zerstörenden Weise gebraucht werden.

Sind wăhrend des Streiks als Folge von vorsătzlichen oder fahrlässigen Handiungen von am Streik beteiligten Arbeitnehmern oder der Gewerkschaft an dem bestreikten Betrieb Schaden aufgetreten, haftet die Gewerkschaft.

In welchen Fällen und in welchen Betrieben Streik und Aussperrung verboten oder aufgeschoben werden können, wird durch Gesetz geregelt.

In den Fällen des Verbots von Streik und Aussperrung oder, wenn sie aufgeschoben sind, am Ende ihrer Aufschiebung wird der Konflikt durch den Hohen Schiedsrichterrat gelöst. In jeder Phase des Konflikts kőnnen die Parteien in gegenseitiger Übereinstimmung den Hohen Schiedsrichterrat anrufen. Die Beschlüsse des Hohen Schiedsrichterrates sind unanfechtbar und gelten als Tarifvertrag. geregelt.

Organisation und Aufgaben des Hohen Schiedsrichterrates werden durch Gesetz

Streik und Aussperrung mit politischem Zweck, Solidaritătsstreik und Solidaritătsaussperrung, Generalstreik und -Aussperrung, Betriebsbesetzung, Arbeitsverzögerung, Herabsetzung der Produktivităt und andere Wiederstandsaktionen sind unzulässig.

Wer an einem Streik nicht teilnimmt, darf durch die Streikteilnehmer von der Arbeit im Betrieb in keiner Weise abgehalten werden." 20

Nach der Darstellung der obigen Bestimmungen der tïrkischen Verfassung möchte ich nun über die Geschichte und den heutigen Stand des Systems und Rechts der kollektiven Arbeitsbeziehungen in der Türkei einige Worte sagen.

Die Gründung der Gewerkschaften war in der Türkei mit dem Gesetz betreffend die Vereinen im Jahre 1938 verboten worden; wurde aber mit dem Gesetz über Gewerkschaften von 1947 wieder frei. Dieses Gesetz gab aber den Gewerkschaften kein Recht, Tarifvertăge im heutigen Sinne zu schließen und Streik auszuüben. Die Gewerkschaften durften jedoch gemäss dem Obligationsgesetz mit Arbeitgebern

${ }^{19}$ Verfassungsgesetz. Artikel 53, zugefügt durch das Gesetz vom 23.7.1995 Nr. 4121.

20 Verfassungsgesetz, Art. 54. 
allgemeine Verträge schließen. Da diese Möglichkeit nicht mit Streikrecht ergänzt wurde, fand das System der allgemeinen Verträge in Praxi keine Verwendung.

Erst nach der Annahme des Verassungsgesetzes von 1961 wurde in der Türkei im Jahre 1963 mit dem Gesetz über die Gewerkschaften (sinngemäß übersetzt über die Berufsverbănde) sowie mit dem Gesetz über Tarifvertrăge, Streik und Aussperrung moglich, Tarifvertrăge im heutigen Sinne zu schließen bzw. Streik und Aussperrung auszuüben. Diese Gesetze wurden mit der Zeit verändert und im Jahre 1983 entsprechend der Verfassung von 1982 durch andere Gesetze mit dem gleichen Titel ersetzt. ${ }^{21}$

In diesen Gesetzen wurden unter anderem die oben angegebenen Betimmungen der Verfassung wiederholt und ergănzt, wie auch diese Gesetze in Form von 1963 die diesbezüglichen Grundsätze der Verfassung von 1961 wiederholten bzw. ergänzten. Die genanten Gesetze regelten im sonsten die Einzelheiten und sonstige Bestimmungen des Rechts auf Berufsverbände (Gewerkschaften, Arbeitgeberverbände und Dachverbande) sowie des Rechts auf Tarifvertrage, Streik und Aussperrung. 22

Seit 1963 funktioniert in der Türkei die Tätigkeit der Berufsverbände sowie das Sytem der kollektiven Arbeitsverhältnisse mit Tarifvertrăgen, Streik und Aussperrung im großen und ganzen recht gut. Das System faßte Wurzel im sozialökonomischen Gebilde und Staatssystem der Türkei. Es bildet heute einen unabdingbaren Bestandteil des demokratisch-politischen Systems und der marktwirtschaftlichen Ordnung der Türkischen Republik.

Selbstverständlich gab es Zeiten, wo sich die Konflikte zwischen den Vertragsparteien sehr verhärteten. Manche Gewerkschaften verhielten sich gesetzwidrig. Sie übten wilde Streike aus. Die Arbeiter besetzten im Einvemehmen mit diesen Gewerkschaften Arbeitsstellen und Betriebe; sogar zerstorten sie manchmal Arbeitsmaschienen und -Gerăte. Diese Gewerkschaften waren meistens linksorientiert oder marxistisch. Sie wurden von solchen politischen, anarchistischen, sogar manchmal von landesfeindlichen Kreisen beeinflusst. Die schwachen Koalitionsregierungen dieser Perioden konnten leider solche gesetzwidrige Verhalten und Ausschreitungen nicht recht vertindem und mußten oft dulden. Auch die Justizbehörden haben oft nicht gemacht, was sie machen sollten.

Manche einschränkende Bestimmungen, Maßnahmen und Sanktionen des Verfassungsgesetzes von 1982, die vielleicht in einer Verfassung als Einzelheit technisch überflüssig sein dürften, weil sie in normalen Gesetzen geregelt werden könnten, sogar schon geregelt waren, ist sicherlich mit diesen Tatsachen zu erklären. Der Gesetzgeber wollte damit vorsichtig sein. Denn gebranntes Kind scheut Feuer.

21 Siehe 2821 sayılı Sendikalar Kanunu (Gesetz für Berufsverbănde Nr. 2821) vom 5.5.1983; 2822 sayılı Toplu Iş Sözleşmeleri Grev ve Lokavt Kanunu (Gesetz für Tarifverträge, Streik und Aussperrung Nr. 2822) vom 5.5.1983.

${ }^{22} \mathrm{Ob}$ die Aussperrung in der türkischen Verfassung ein Recht bildet, wird allerdings diskutiert. Tatsächlich wird in der Verfassung im Gegensatz zu Streik bei der Aussperrung das Wort "Recht" nicht verwendet. Aber die Grundsätze der Anwendung der Aussperrung wird darin genau so geregelt oder einem Gesetz uberlassen wie beim Streik. Deshalb bildet nach unserer Meinung auch die Aussperrung ein Verfassungsrecht. Aber das Streikrecht hat vor dem Recht Ausperrung Priorität. 
All die oben erwăhnten Ereignisse bildeten jedoch extreme Situationen und passienten entsprechend dem damaligen Weltbild zum Ende der sechziger Jahre und in den siebziger Jahren. Nur wurde es damit in der Türkei recht übertrieben.

Folgende Tabelle enthält Zahlen über Anwendung des Rechts auf Tarifverträge und Streik sowie die verlorenen Arbeitstage infolge der Streike.

Tarifvertrăge und Streike

$\begin{array}{ccccc}\text { Jahre } & \begin{array}{c}\text { Zahl der } \\ \text { Tarifverträge }\end{array} & \begin{array}{c}\text { Zahl der erfaßten } \\ \text { Arbeiter }\end{array} & \begin{array}{c}\text { Zahl der } \\ \text { Streike }\end{array} & \begin{array}{c}\text { Zahl der verlorenen } \\ \text { Arbeitstage }\end{array} \\ 1963 & 96 & 9000 & 8 & 19737 \\ 1970 & 1516 & 551000 & 72 & 220189 \\ 1980 & 1813 & 3300001 & 220 & 1303252 \\ 1984 & 1185 & 340095 & 4 & 4947 \\ 1985 & 2721 & 919810 & 21 & 194296 \\ 1990 & 1954 & 483852 & 458 & 3466550 \\ 1993 & 3809 & 1068289 & 49 & 574741 \\ 1995 & 2357 & 765928 & 1210 & 4838241\end{array}$

Zusammengestellt nach den Tabellen von "Arbeitsstatistiken" des Ministeriums fur Arbeit und Soziale Sicherheit, Ankara Juni 1996.

Wie auch aus den oben zitierten Bestimmungen des Verfassungsgesetzes zu verstehen ist, herrscht in der Türkei hinsichtlich der Gewerkschaften und Arbeitgeberverbände eine absolute Freiheit. Die Gründung dieser Verbände ist sehr einfach und ohne vorherige Erlaubnis frei. Ihre Schließung ist nur im Falle der Gesetzwidrigkeit möglich und wird vom Gericht beschlossen. Der Eintritt und Nichteintritt in diese Berufsverbănde sowie der Austritt aus ihnen sind frei. Bei der Einstellung und Beschäftigung von Arbeitnehmern darf weder eine allgemeine und noch eine beschränkte Organisationsklausel durchgeführt werden, mit angelsächsischem Terminus ausgedrücǩt closed shop-clause und union shop-clause sind verboten. Dies alles zeigt, daß in der türkischen Verfassung Koalitionsfreihcit sowohl im positiven als auch im negativen Sinne gewăhrt ist.

Die Ziele der Gewerkschaften und Arbeitgeberverbănde sind allerdings beschränkt auf den Schutz und die Entfaltung der wirtschaftlichen und sozialen Interessen ihrer Mitglieder in Arbeitsbeziehungen. Politische Tätigkeit und die Beziehung zu politischen Parteien sind ihnen verboten.

Nach Türkischer Verfassung "durften die Gewerkschaften und Arbeitgeberverbände bis 1995 keine politischen Ziele verfolgen, sich politisch nicht betätigen, von politischen Parteien nicht unterstützt werden und diese nicht unterstützen, zu diesen 
Zwecken mit Vereinen, berufsständigen Vereinigungen mit der Natur von Körperschaften des $b$ ffentlichen Rechts und Stiftungen gemeinsam nicht tătig werden. ${ }^{.23}$

Diese einschränkenden Bestimmungen der Verfassung wurden auch im Gesetz über Gewerkschaften (sinngemäss übertragen Gesetz über Berufsverbände) ${ }^{24}$ wiederholt sowie in einigen Punkten ergănzt.

Der Artikel der Verfassung, der diese Einschränkungen brachte, wurde jedoch mit einer Änderung im Jahre 1995 aufgehoben. Die Bestimmungen des Gesetzes über Gewerkschaften und somit die Verbote hinsichtlich der politischen Tătigkeit, bzw. politischer Beziehungen blieben jedoch weiter in Gültigkeit. Nach diesem Gesetz gelten allerdings die Tătigkeiten und Manifeste der Mitglieder von Berufsverbănden mit dem Zweck zum Schutz und zur Entfaltung ihrer wirtschaftlichen und sozialen Interessen nicht als politische Tätigkeit.

Andererseits wurden die Einschränkungen und Verbote hinsichtlich der politischen Tătigkeit der Gewerkschaften und Arbeitgeberverbănde praktisch oft nicht beachtet. Die gesetzwidrigen Verhalten von Gewerkschaften und Arbeitgeberverbänden im Hinblick ihrer politischen Tătigkeit wurden von verantwortichen Stellen geduldet. .

Die Gewerkschaften und Arbeitgeberverbände sind in der Türkei wie in Deutschland nach Industrieprinzip organisiert. Eine Organisierung nach Berufs-bzw. Fachverbandsprinzip ist nicht möglich. Im Gesetz über Gewerkschaften wurden zwischen 28 Industriebereichen unterschieden. Die Dachverbände dürfen nur in Form einer Konfederation gebildet werden. Es dürfen mehrere Dachverbände gegründet werden. Zur Zeit gibt es vier Dachverbände der Gewerkschaften und ein Dachverband der Arbeitgeberverbände. Arbeitnehmer und Arbeitgeber dürfen zur gleichen Zeit nur einem Berufsverband und dic Berufsverbände einem Dachverband Mitglied werden.

Die Gewerkschaften und Arbeitgeberverbände haben voneinander unabhänging zu sein. Die Mitglieder dieser Verbände dürfen nicht zugleich Mitglied des gegnerischen Verbandes sein.

Die Gewerkschaften haben sich, wie schon oben erwăhnt, im türkischen politischen, wirtschaftlichen und sozialen System eingebürgert. Zwischen 1984 und 1996 schwankte der Anteil der Gewerkschaftsmitglieder an der gesamten Arbeiterzahl, die das Gesetz über Gewerkschaften erfaßt, zwischen 53-69 \%. Dieser Anteil betrug im Januar 1996 67,84 \%. Es gibt allerdings viele schwarz Arbeitende, deren Zahl nicht leicht zu schätzen ist. Diese würden auf diese Ziffern sinkend wirken.

In der Türkei dürfen die Tarifverträge nur zwischen Gewerkschaften und Arbeitgeberverbänden oder einzelnen Arbeitgebern, die kein Mitglied von Arbeitgeberverbänden sind, geschlossen werden. Die Gewerkschaften müssen dazu mindestens $10 \%$ der Arbeiter ihres Industriebereiches und mehr als die Hälfte der Arbeiter

${ }^{23}$ Verfassungsgesetz, Artikel 52, abgeschafft durch das Gesetz vom 23.7.1995, Nr. 4121.

${ }^{24}$ In der Türkei versteht man unter Gewerkschaften die Arbeitergewerkschaften und Arbeitgeberverbände zugleich. 
von Werken oder Betrieben, wo Tarifverträge geschlossen werden sollen, als Mitglied haben.

Bei Konfliktfällen darf der Streik, bzw. die Aussperrung erst dann erklărt werden, wenn ein obligatorisch einschaltender Schlichtungsprozess nicht zur Lösung des Konfliktes geführt hat.

Wenn die Gewerkschaft als gegnerischer Verhandlunspartner kein Streik erklărn hat, darf auch die Arbeitgeberseite keine Aussperrung erklären.

Die Arbeitgeber dürfen in der Türkei, wăhrend des Streikes, bzw. der Aussperrung anstelle der Arbeiter, deren Arbeitsverhaltuis suspendiert wurde, in keiner Weise neue Arbeiter einstellen. Dagegen dürfen auch diese Arbeiter während des Streikes oder der Aussperrung anderswo nicht arbeiten.

Diese Regelung ist eine Sonderheit des türkischen Systems. Eine solche Einschränkung trifft man in anderen Ländern nicht. Da es in der Türkei eine Arbeitşlosigkeit in höherem Maße gibt, wollte man damit die schwächere Position der arbeitnehmerseite bei kollektiven Arbeitsbeziehungen stärken, indem man die Effizienz des Streikes erhohte. Sonst würde der Streik in der Türkei unter der Bedingung der hohen Arbeitslosigkeit kein wirksames Kampfmittel gegen Arbeitgeber bilden. Um dies förmlich auszugleichen, wurde, wie erwăhnt, auch die Arbeit der streikenden oder ausgesperten Arbeiter verboten.

Andererseits dürfen auch die Arbeiten der streikenden Arbeiter wăhrend des Streiks von am Streik nicht beteiligten Arbeitern des Arbeitgebers nicht fortgeführt werden. Mit dieser Regel ist ebenfalls die Verstärkung der Stellung der Arbeitnehmer bezweckt.

Wenn beim Streik sowic bei der Aussperrung der im Gesetz vorgesehene ProzeB nicht eingehalten oder gegen Verbote des Gesetzes verstoßen wird, sind der Streik bzw. die Aussperrung gesetzwidrig oder mit anderen Worten sind sie wild. Wilder Streik und wilde Aussperrung sind nicht erlaubt.

Ausserdem darf der Streik nur seitens der Gewerkschaft, die die Verhandlungen für den Tarifvertrag geführt hat, erklärt werden. Die Arbeitnchmergruppen dürfen keinen Streik erklären bzw. ausüben.

Nun wollen wir kurz erklären, welche Verbote für Streik und Aussperrung im Gesetz vorgeschen sind:

Bei bestimmten Arbeiten und Umständen sind Streik und Aussperrung nicht erlaubt: Das sind 1) Rettungsarbeiten; 2) Bestattungs-und Beerdigungsarbeiten; 3) Wasserversorgung, Strom-und Gaserzeugung, Braunkohlenförderung für thermische Zentrale; Sondage-, Förderungs-, Rafinierungs- und Verteilungsarbeiten von Erdgas und Erdర1, sowie petrochemische Arbeiten, bei denen die Produktion mit Naphtha oder Erdgas beginnt; 4) Dienste bei Banken und Notaren; 5) Feuerwehr, Personentransportdienste innerhalb der Städte im Wasser, auf Landstraßen und Schienen.

Bei bestimmten Produktions-und Dienststătten sind Streik und Aussperrung verboten: Das sind 1) Produktionstätte von Impfstoff und Serum; Heilanstalten wie 
Krankenhäuser, Kliniken, Sanatorien, Präventorien, Kinderkrankenhäuser und Apotheken; 2) Bildungsstătte, Kinderpflegeanstalten und Altersheime; 3) Friedhöfe; 4) Betriebe, die vom Verteidigungsministerium, von der Generalkommandantur für Gendarmerie und der Kommandantur für Sicherheit der Meereskiusten unmittelbar betrieben werden.

Bei Kriegs- und allgemeinen oder teilweisen Mobilisationszustanden sind Streik und Aussperrung verboten. Bei Katastrophen wie Feuer, Hochwasser, Erdrutschen, Lawinen oder Erdbeben, die das allgemeine Leben lahmlegen, kann der Ministerrat in notwendig erachteten Betrieben oder Industriebereichen in Katastrophengebieten für die Dauer dieser Zustände Streik und Aussperrung verbieten.

Bei Beforderungsmittel im Meer, Land und in der Luft, die ihren Zielort noch nicht erreicht haben, sind Streik und Aussperrung verboten.

Außer diesen Verboten gibt es noch die hinsichtlich der Bestimmungen der Verfassung oben angegebenen Verbote für Streikfälle und ăhnliche Verhalten der Arbeitnehmer sowie für Aussperrungen.

Wenn ein Streik oder eine Aussperrung allgemeine Gesundheit oder nationale Sicherheit gefăhrdet, darf vom Ministerrat für 60 Tage aufgeschoben werden. Gegen diesen Beschluß des Ministerrates kann beim Hohen Verwaltungsgericht Einspruch erhoben werden. Wăhrend der Aufschicbungszeit hat der Arbeitsminister mit Hilfe eines beauftragten Schlichters von der offiziellen Schlichterliste alle Anstrengungen zu zeigen, um den Konflikt beizulegen. Im Falle der Nichtbeseitigung des Konflikts in der Aufschiebungszeit, hat der Hohe Schiedsrichterrat darüber zu entscheiden.

Auch in den Fällen des Verbots von Streik und Aussperrung wird der Konflikt ebenfalls durch den Hohen Schiedsrichterrat gelost, falls sich die Vertragspartner nicht verstăndigen können.

\section{Gewährleistung der Lohngerechtigkeit}

Nach türkischer Verfassung "hat der Staat die notwendigen Maßnahmen zu treffen, damit die Arbeitehmer einen ihrer Arbeit angemessenen, gerechten Lohn erhalten und in den Genuß der sonstigen Sozialleistungen kommen.

Bei der Feststellung des Mindestlohns wird die wirtschaftliche und soziale Lage des Landes berücksichtigt " 25

Zur Verwirklichung des Grundsatzes, Arbeitnehmern einen ihrer Arbeit angemessenen gerechten Lohn zu gewähren, gehören vor allem die Maßnahmen zur Entwicklung der türkischen Wirtschaft sowie zur Erhöhung ihrer Produktivităt. Auch die Maßnahmen zur Förderung bzw. zur Regelung der Anwendung des Rechts auf Gewerkschaften, Tarifverträge und Streik, um die Verhandlungsposition der Arbeitnehmer gegenüber Arbeitgebern zu stărken, gehören dazu.

${ }^{25}$ Verfassungsgesetz, Artikel 55. 
Es ist nicht moglich, hier im Rahmen dieses Artikels die Anstrengungen zur Entwicklung der türkischen Wirtschaft darzulegen. Was die Gewerkschaften und Tarifverträge betrifft, wurde schon oben darüber Erklärungen gemacht.

Die weiteren Instrumente zur Verwirklichung dieses Grundsatzes der Verfassung bilden die Steuerpolitik und Mindestlohne. Die direkten Kontrolle und Interventionen über eigentliche Lơhne sind mit marktwirtschaftlicher Ordnung der Türkei nicht zu vereinbaren. Eine solche Politik wurde auch nicht ausgeübt. Der Staat kann in dieser Beziehung nur die Gehălter seiner Beamten bestimmen und das tut er auch. Damit wird allerdings nicht unbedingt gesagt, $\mathrm{da} B$ die Beamten stets ein ihrer Arbeit angemessenes gerechtes Gehalt erhalten.

Wie in jedem Land gibt es auch in der Türkei progressive Einkommenssteuer. Der steuerfreie Anteil des Arbeitseinkommens ist sehr niedrig festgesetzt. Von sozialgesinnten Kreisen sowie von Seiten der Arbeitnehmer wird gefördert, daß der dem Mindestlohn entsprechenden Teil des Arbeitseinkommens steuerfrei sein soll. Bisher konnte jedoch diese Forderung nicht erfüllt werden.

Andererseits ist in der Türkei der Anteil der indirekten Steuern an den gesamten Steuereinnahmen relativ groß. Ebenfalls ist die Steuerhinterziehung in den Kreisen der Nichtarbeitnehmer zu hoch. Unter diesen Umständen ist man in der Türkei weit davon entfernt, den diesbezüglichen Grundsatz der Verfassung verwirklicht zu haben.

Die Mindestlöhne werden mit Hilfe einer Sonderkommission vom Arbeitsministerium spätestens alle 2 Jahre festgesetzt. In dieser Kommission sind der Staat, Arbeitnehmer und Arbeitgeber mit je fünf Mitgliedem paritătisch vertreten.

In folgender Tabelle sind die Entwicklung der Mindestlohne sowie die Erhőhung der Preise vergleichend dargestellt:

Jahre

1983

1984

1985

1986

1987

1988

1989

1990

1991

1992

1993

1994

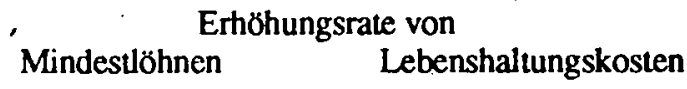

0

62

37,1

49,7

51,4

44,2

0

30,7

68,8

55,1

79,3

75,2

69,7

68,8

78,6

60,6

84,0

71,1

93,5

67,9

80,9

71,3

72,4 
*) Schătzung

Quelle: Arbeitsstatistiken vom Ministerium für Arbeit und Soziale Sicherheit, Ankara Juni 1996, s. 150; Verschiedene Monats-und Jahresbulletins des Staatlichen Statistikinstituts sowie die von diesem Institut persönlich erhaltenen Zahlen.

Über die eigentlichen durchschnitulichen Lohne haben wir in der Türkei keine zuversichtlichen Zahlen. Es gibt zwar Zahlen über die Löhne in der verarbeitenden Industrie. Diese sind aber auch nicht sehr zuverlässig. Wir wissen natürlich, daB die Lơhne in der Türkei im Vergleich zu europäischen Landern sehr niedrig sind. Der Grund dafür liegt an dem niedrigen Niveau des Sozialproduktes, der schlechten Verteilung des Volkseinkommens sowie an der großen Arbeitslosigkeit in der Türkei. Aber manche starken Gewerkschaften sichem ihren Mitgliedern Löhne, die den durchschnituslohn weit übertreffen.

Bei einem Vergleich mit Löhnen in Europäischen Ländern durch Welchselkurse würden türkische Löhne noch schlechter ausfallen als sie sind. Wenn man als Vergleichsmaß die Kaufkraftsparităten nehmen würde, würden die türkischen Lơhne mindestens Zweifache höher ausmachen.

\section{Gesundheit und Umwelt}

Die türkische Verfassung bringt auch Bestimmungen über Gesundheitsdienste und Umweltschutz. Jedermann hat danach "das Recht auf Leben in einer gesunden und ausgeglichenen Umwelt.

Die Entwicklung der Umwelt, die Gewăhrleistung einer gesunden Umwelt und die Verhinderung der Umweltverschmutzung sind die Pflicht des Staates und der Bürger.

Um eine Lebensführung von jedermann in körperlicher und geistiger Gesundheit zu gewăhrleisten und unter Erhöhung der Wirtschaftlichkeit und Produktivităt menschlicher und materieller Kraft die Zusammenarbeit der Gesundheitseinrichtungen zu verwirklichen, plant der Staat die Gesundheitseinrichtungen einheitlich und regelt ihre Dienstleistungen.

Der Staat erfüllt diese Aufgabe, indem er die Gesundheits-und sozialen Organisationen im offentlichen und privaten Sektor nutzt und kontrolliert.

Um die Gesundheitsfürsorge in aller Breite durchzuführen, kann durch Gesetz eine allgemeine Krankenversicherung gegründet werden."26

Maßnahmen zum Umweltschutz sind relativ neu in der Türkei. Klärung der Abwässer und Behinderung der Luftverschmutzung sind relativ neue Begriffe. Seit den, achtziger und besonders neunziger Jahren wird jedoch über diese Themen viel gesprochen.

${ }^{26}$ Verfassungsgesetz, Art. 56. 
Es wurde auch manches getan. Viele Projekte werden auch langsam in die Tat versetzt. Die Luft von Ankara wurde mit Hilfe der Erdgasheizung ziemlich gesăubert. In Istanbul und einigen anderen Stădten gibt es dafür Bestrebungen. Ebenso werden in mehreren Städten und turistischen Ortschaften Klärungsanlagen gebaut oder deren Bau befindet sich bei Planung. Auch die Industrie bemüht sich danach, Abgase zu vermindern und Abwässer zu klären. Man befindet sich aber dabei ganz am Anfang. Es muß in diesem Gebiet viel getan werden. Es ist aber dafür ein Bewußtsein schon da. Die offentliche Weltmeinung und Anstrebungen für Export spielten zweifellos im Erwecken dieses Bewußtseins mit eine Rolle.

In der Türkei werden die Walder infolge der Brände und Abholzung immer weniger.

Seit Anfăngen der Republik wird in der Türkei bestrebt, die Gesundheit des Volkes zu sichern. Die Massenkrankheiten wie Malaria, Tuberkulose, Lepra und Trachom wurden erfolgreich bekämpft und werden zum Teil noch bekämpft. Man hat bestrebt die Zahl der Ärzte und Hilfspersonen sowie die Zahl der Krankenhäuser und Krankenbetten zu erhöhen. In den letzteren Jahren erhöhten sich neben offentlichen auch die Zahl der privaten Krankenhäuser und Kliniken. Heute fallen in der Türkei pro Arzt etwa 900 und pro Krankenbett rund 400 Personen an. Diese Ziffern werden zweifellos im Vergleich zu europäischen Ländern zu hoch scheinen. Sie dürften aber im Vergleich zu früheren Zeiten der Türkei befriedigend sein. Ebenso ist die Sterberate von Baybs ständig gesunken. Heute beträgt sie durchschniulich etwa $44 \%{ }^{27}$

In den staatlichen Kliniken und Krankenhăusern werden die Mittellosen kostenlos behandelt. Seit den 90 er Jahren werden dazu den Armen sogenannte grüne Karten erteilt. Mit dieser Karte erhält man das Recht, in staatlichen Krankenhäusern kostenlos oder auf Kosten des Staates behandelt zu werden. Natürlich haben nicht alle Bedürftigen die Chance, von dieser Möglichkeit Gebrauch zu machen, weil die Mittel des Staates begrenzt sind. Andererseits entstanden schon bei der teilweisen Ausnutzung dieser Möglichkeit große finanzielle Probleme in den staatlichen Krankenhăusern und Universitătskliniken. Denn der Staat kann die Rechnungen nicht rechtzeitig oder gar nicht begleichen.

Seit langem befindet sich die Errichtung einer allgemeinen Krankenkasse in Tagesordnung. Aber dazu kam es noch nicht. Bis dahin müssen auch viele Probleme gelठ̋t werden.

\section{Recht auf Wohnung}

Die türkische Verfassung gibt dem Staat auch in diesem Bereich Aufgaben. Danach "trifft der Staat im Rahmen einer Planung, welche die Besonderheiten der Städte und die Umweltbedingungen berücksichtigt, die Maßnahmen zur Befriedigung des Wohnunsbedarfs. Er unterstützt außerdem die Unternehmungen des sozialen Wohnungsbaus." 28

${ }^{27}$ Fur die Zahlen vgl. Staatliches Planungsamt, Jahresprogramm 1996, a.a. 0. S. 30 ff. ${ }^{28}$ Verfassungsgesetz, Art. 57. 
Die türkische Bevolkerung vermehrt sich sehr schnell. Deshalb herrscht in der Türkei stets Mangel an Wohnungen. Außerdem gibt es große Einwanderungen vom Land in die Stădte. Dadurch wird der Bedarf an Wohnungen noch mehr erhöht.

Eine staaliche Bank gibt seit jeher den wohnungslosen privaten Personen und Genossenschaften Kredite für Wohnungsbau bzw. für Ankauf von neugebauten Wohnungen. Sie baut auch selber Wohnungen in den Städten bzw, in den Satellitenstädchen und verkauft diese der Bevollkerung auf Kredit.

Seit den 80 er Jahren gibt es auch eine zu diesem Zweck ins Leben gerufene staatliche Organisation. Sie erteilt den Baugenossenschaften Kredite und baut auch selber Wohnviertel und Satellitenstädtchen, deren Wohnungen wiederum der Bevolkerung auf Kredit verkauft werden. Zur Finanzierung werden bestimmte Staatseinnahmen auf den Fonds dieser Organisation direkt übertragen. Allein diese Organisation hat zwischen 1984- (September) 1994 für 908.339 Wohnungen Kredite gewăhrt.

$\mathrm{Da}$ aber die finanziellen Quellen von beiden begrenzt sind und dazu infolge der hohen Inflationsrate der RückfluB der Kredite, reell gesehen, nicht in vollem Umfang geschieht, gingen die Zahl und reale Höhe der von diesen Anstalten gewăhrten Kredite sowie ihre Bautătigkeiten in den letzten Jahren erheblich zurück. Unter heutigen Verhăltnissen bilden diese Kredite keinen erwăhnenswerten Bruchteil der Bau- bzw. Ankaufskosten, wenn sie überhaupt gegeben werden.

Daneben bauen auch die privaten Bauunternehmer Wohnhăuser und verkaufen diese als Eigentumswohnungen und Villen den kaufkräftigeren Nachfragem.

Da alle diesen Angebote den Bedarf nicht decken, herrscht auf diesem Markt ein bemerkenswerter Nachfrageïberhang.

Die Wohnungslücke wird zum Teil durch den Bau von Elendshăusem gefullt. Die Einwanderer in die großen Stădte aus dem Land bauen an den Răndern der Stădte, manchmal in sehr schönen Ortschaften ihre einfachen Häuser. Im Laufe der Zeit entwickeln sich diese oft zu hässlichen Etagenwohnungen. Diese Tendenz steigert sich auch mit der Zeit. So entstehen dadurch planlos, informell und illegal aus dem Boden geschossene Elendsviertel bzw. Slums. ${ }^{29}$ Die Grundstücke dafür bilden meist Staatsgelănde. Diese werden oft von der Mafia planlos parzelliert und diesen Leuten verkauft. Die Gemeinden dulden diese Entwicklung. Sie fördem diese Entwicklung sogar dadurch, daß sie diesen Hăusern Strom, Wasser und Gas bringen bzw. ihre engen Schmutzgassen pflastern, weil sie bei den Wahlen ihre Stimmen gewinnen wollen. Auch die bestechlichen Beamten der Gemeinden leisten zur Entwicklung dieser Elendsviertel besonders bei ihrer Entstehung ihren Beitrag.

Andererseit fördern auch die Politiker diese Entwicklung dadurch, daß sie von Zeit zu Zeit durch Gesetzerlasse als Wahlgeschenkè diese Elendhăuser legalisieren und ins Grundbuch eintragen lassen.

${ }^{29} V_{\text {gl }}$. S. Dilik, Wirtschaftliche Entwicklung und Strukturwandel in der Türkei, in: A.0. Siyasal Bilgiler Fakultesi Dergisi (Zeitschrift der Staatswissenschaftlichen Fakultăt der Uni. Ankara), Bd XLV. Nr. 1-4, 1990, S. 39-52. 
Manche von diesen Vierteln, deren Hăuser sich noch nicht zu großen hăsslichen Etagenhäuser entwickelt haben, werden durch neuc Parzellierung und Planung seitens der Gemeinden saniert. Die Grundstücke werden von den Besizzem gegen eine oder einige Eigentumswohnungen den Bauunternehmern zur Verfügung gestellt. Diese verkaufen die weiteren Wohnungen an Dritte. Somit wird die Stadt verschönert. Aber die Besitzer der illegal und meist auf Staatsgelände gebauten Hăuser machen daraus ein nicht zu unterschätzendes unbergründetes Vermögen.

Seit einiger Zeit gibt es in manchen Orten, wie z.B. in Istanbul, Izmir und Antalya Anstrengungen gegen weitere Ausbreitung von Elendsvierteln. Die neugebauten Slumshäuser werden abgerissen. Diese Entwicklung bedeutet jedoch in keiner Weise eine allgemeine Änderung der Gesinnung bzw. der Politik von Verantwortlichen, sondern hăngt von der persönlichen Haltung der Bürgermeister von diesen Orten ab.

\section{Soziale Sicherheit ${ }^{30}$}

Nach türkischer Verfassung "hai Jedermann das Recht auf soziale Sicherheit. Der Staat trifft die notwendigen Maßnahmen zur Gewährleistung dieser Sicherheit und begründet hierzu die notwendige Organisation." 31

Die Sozialversicherung im modemen Sinne wurde in der Türkei erst im Jahre 1946 gegründet, wenn auch ihre Vorläufer noch etwas zurückgehen.

Schon im Arbeitsgesetz von 1936 wurde die Gründung vorgesehen und Grundsătze dafür festgelegt. Der zweite Weltkrieg verzögerte jedoch die Gründung. Erst 1945 konnten dann das Versicherungsgesetz für Arbeitsunfälle, Berufskrankheiten und Mutterschaft sowie das Gesetz über Arbeiterversicherungsanstalt erlassen werden. Die Gründung und Tătigkeit erfolgte 1946. Mit der Zeit wurde auch andere Versicherungsbereiche gegründet und die Organisation nahm den Namen "Sozialversicherungsanstalt" an. Im Jahre 1971 wurde dann Sozialversicherungsanstalt für die unabhängig Arbeitenden gegründet.

Der Kreis der erfaßten Personen verbreitete sich ebenfalls.

Heute bestehen in der Türkei mit Ausnahme von Arbeitslosen- und Pflegeversicherung alle Versicherungsbereiche. Mit anderen Worten werden die Gefahren wie Krankheit, Unfälle, Mutterschaft, Invaliditäh, Alter und Tod gedeckt. Kindergeld wird in der Türkei nicht gezahlt. ${ }^{32}$

${ }^{30}$ Zur sozialen Sicherheit in der Türkei siche S. Dilik, Türkiye'de Sosyal Sigortalar-lktisadi Açıdan Bir Tahlil Denemesi (Die soziale Sicherung in der Türkei - Versuch einer volkswirtschaftlichen Analyse), Ankara 1972; Sosyal Güvenlik (Soziale Sicherung), Ankara 1992; Erken Emeklilik (Fruhpensionierung); in: Işveren (Arbeitgeber), Bd. XXX, Nr. 5 Februar 1992; S. 9-13; Probleme bei der Angleichung der sozialen Sicherung der Turkei an die soziale Sicherung der EG, in: Dic Türkei auf dem Wege in die EG, hrsg. v.W. Gumpel, Oldenbourg, s. 105-117.

31 Verfassungsgesetz, Art. 60.

32 Den Beamten wird zwar für Kinder einen Zuschlag zu ihrem Gehalt bezahlt. Die Höhe dieser Zahlung ist aber so niedrig, daß man nicht davon sprechen könnte, daß die Beamten Kindergeld bekommen. 
Es gibt in der Türkei drei Hauptanstalten für soziale Sicherung: die Sozialversicherungsanstalt für die Arbeitnehmer, die Sozialversicherungsanstalt für die unabhängig Arbeitenden und die Pensionskasse für Beamten. Außerdem gibt es separate Anstalten für die Beschäftigten von Kammern, Banken, Börsen, Versicherungsgesellschaften und ihren Dachorganisationen usw. Alle diese Gruppen werden mit manchen Ausnahmen gegen die oben erwăhnten Risiken geschützt. Gegen Manche von diesen Risiken werden die Beamten vom Staat als Arbeitgeber geschützt.

Heute befindet sich etwa 80 Prozent der Bevollkerung als Versicherte, Beamte, Rentner oder als Kinder, Ehepartner und die Eltern von diesen unter dem Schirm der sozialen Sicherheit. Im Hinblick auf Gesundheitshilfe sind jedoch nur rund $65 \%$ der Bevolkerung unter dem Schutz der sozialen Sicherung, weil die Anwendung der Krankenversicherung begrezter ist. Die unabhänging Arbeitenden in der Landwirtschaft unter 22 Jahren sowie undauerhafte Arbeitnehmer in der Landwirtschaft konnten nicht unter den Schutz der sozialen Sicherheit genommen werden. Diese konnen jedoch gegen bestimmte Gefahren freiwillig versichert werden, wenn sie mindestens 18 Jahre alt sind. Diese beiden Gruppen können gegen die Gefahr Krankheit und die undauerhaft Arbeitenden in der Landwirtschaft noch dazu gegen Unfalle und Berufskrankheiten nicht versichert werden.

Die sozialen Versicherungsanstalten befinden sich in der Türkei zur Zcit in einer großen Finanzkrise. Es gibt natürlich vicle Gründe dafür. Aber den Hauptgrund bildet die Auflockerung der Voraussetzungen zur Gewăhrung der Altersrenten. In der Türkei können Frauen mit 38 und Männer mit 43 Jahren pensioniert werden, bzw. Altersrente bekommen, falls sie seit 20 Jahren als Frau und 25 Jahren als Mann versichert sind und schon 5000 Tage Rentenversicherungsprämie bezahlt haben. Diese Erleichterungen haben natürlich mit dem Begriff sozialer Sicherheit nichts zu tun. Sie wurden aus politischen Überlegungen im Jahre 1969 eingeführt 1985 wurden sie in der Regierunsperiode von Özal unter Beachtung von bestimmten erworbenen Rechte Stufenweise abgeschaff. Sie wurden 1992 als Wahlgeschenk leider wieder eingeführt. Nach den heute geltenden Bestimmungen der betreffenden Gesetze dürfen die Rentner noch dazu nach ihrer Pensionierung, d.h. während sie Altersrente bekommen, ihre Arbeit fortführen oder in einer anderen Stelle arbeiten. Nur die Beamten dürfen ihre Arbeit als Beamter nicht fortuihren.

Auf diese Weise wurde die soziale Sicherung in der Türkei in diesem Hinblick entartet. Neuerdings möchte man wieder die genannten Erleichterungen abschaffen. Dazu wurden sogar Gesetzesvorlagen vorbereitet. Aber die politischen Überlegungen verzögern die Verwirklichung dieses Vorhabens.

Für die im Hinblick auf dic soziale Sicherheit besonders Schutzbedürftigen bringt die türkische Verfassung besondere Bestimmungen. Danach "schützt der Staat die Witwen und Waisen der im Krieg und bei Erfüllung ihrer Pflicht Gefallenen, die Kriegsinvaliden und Veteranen und sorgt für einen angemessenen Lebensstandard für sie in der Gemeinschaft."

Der Staat trifft die Maßnahmen zur Gewährleistung des Schutzes der Behinderten und zu ihrer Eingliederung in das Gemeinschaftsleben. 
Die Alten werden vom Staat geschlitzt. Die staatliche Hilfe und die anderen zu gewăhrenden Rechte und Erleichterungen für die Alten werden durch Gesetz geregelt.

Der Staat trifft Maßnahmen aller Art, um die schutzbedürftigen Kinder der Gemeinschaft zuzuführen.

Er gründet die zu diesen Zwecken notwendige Organisation und Einrichtungen oder LaBt sie grinden." ${ }^{33}$

Diese Bestimmungen der türkischen Verfassung sind vielmehr für den Bevolkerungsteil gedacht, der normalerweise ausserhalb des Systems der Sozialversicherung steht. Es handelt sich hier um die MaBnahmen für soziale Fürsorge.

Diese Ziele der Verfassung konnten leider nicht in vollem Umfang in die Tat versetzt werden.

Nur einem kleinen Teil von erwăhnten Gruppen wird z.B. Sozialeinkommen gewăhrt. Andererseits ist die Höhe der gewährten Sozialeinkommen auch für eine bescheidene Lebensführung unzureichend. Die Witwen und Waisen der im Krieg oder bei Erfüllung ihrer Pflicht gefallenen, die ohnehin eine normale Rente bekommen, erhalten dagegen einen Zuschlag dafür.

Für die Alten über 60 Jahre gibt es bei staatlichen Eisenbahn- und Luftlinien. sowie bei offentlichen städtischen Transportmitteln Ermäßigungen.

Gemå einem 1976 erlassenen Gesetz wird den bedürtigen Alten über 65 Jahre eine Art Rente gewăhrt. Jedoch ist die Höhe dieser Rente heute so niedrig, daß man sie symbolisch nennen darf. Diese Höhe war auch am Anfang nicht ausreichend.

Die Verantwortung für die Dienste zum Schutze der hilfsbedürftigen Kinder und Alten trägt die Anstalt für soziale Dienste und Kinderschutz. Die Zahl der Kinder- und Jugendheime ist allerdins im Vergleich zum Bedarf sehr gering. Die Kinder werden auch bei Pflegefamilien untergebracht und dort unterstützt. Dies alles reicht nicht aus, um alle hilfsbedürftigen Kinder zu schützen. Der große Teil der schutzbedürftigen Kinder ist deshalb vielmehr ihrem Schicksal auf der Straße überlassen.

Ebenso ist die Zahl der Altersheime unzureichend. Ihre Qualităt lăßt, abgesehen von einigen neuen privaten Heimen, meist viel zu wünschen ubrig. Die Gründung von Altersheimen in modemem Sinne ist in der Türkei relativ neu.

Die Behinderten werden auch nicht genügend geschützt. Zur Erleichterung ihres tăglichen Lebens wurden vom Staat bisher kaum Maßnahmen getroffen.

Zur Beschäftigung der Behinderten wurde jedoch im Arbeitsgesetz Betimmungen gebracht. Danach müssen Arbeitgeber, die über 50 Arbeiter beschäftigen, zwei ihrer Arbeitnehmer aus den Kreisen von Behinderien wăhlen. Sie müssen den behinderten

${ }^{33}$ Verfassungsgesetz, Art. 61. 
Arbeitern ihrer korperlichen und geistigen Lage angemesene Arbeiten geben. Ähnliche Bestimmungen gibt es im Arbeitsgesetz auch fur die Vorbestraften.

Für den Bevölkerungsteil, der von Anstalten der sozialen Sicherheit nicht oder nur teilweise erfaßt wird, bestand im System der sozialen Sicherung trotz oben erwähnten Fürsorgemaßnahmen eine große Lücke. Um diese Lücke zu füllen, wurde 1986 "das Gesetz zur Förderung der gegenseiten Hilfeleistung und Solidarităt" erlassen. Nach diesem Gesetz werden Betrăge als bestimmte Bruchteile von manchen Staatseinnahmen in den zu diesem Zweck gebildeten Fonds übertragen. Die gemäB diesem Gesetz in Bezirken und Kreisen gebildeten Stiftungen verteilen die Mittel dieses Fonds an die Bedürftigen als soziale Hilfe. ${ }^{34}$ Die Mittel des Fonds werden aber leider auch außerhalb der Ziele dieses Gesetzes angewendet und zum Teil auf die Staatskasse übertragen.

Trotz obigen Maßnahmen kơnnen in der Türkei nicht allen Befürftigen geholfen werden. Der Hauptgrund dafür liegt zweifellos in der Unzulänglichkeit der Hilfsquellen des Landes.

\section{Jugend und Sport}

Türkische Verfassung hat auch Bestimmungen zum Schutz der Jugend und zur Entwicklung des Sports. Sie lauten wie folgt:

"Der Staat trifft die Maßnahmen zur Gewăhrleistung der Entwicklung und Erziehung der Jugend, welcher unsere Unabhängigkeit und unsere Republik anvertraut sind, im Lichte der Naturwissenschaft, im Sinne der Prinzipien und Reformen Atatürk und gegen Anschaungen, welche die Aufhebung der Unteilbaren Einheit von Staatsgebiet und Staatsvolk-zum Ziel haben.

Der Staat trifft die notwendigen Maßnahmen, um die Jugendlichen vor Alkoholismus, Betăubungsmittcln, Kriminalităt, Glücksspiel und ăhnlichen schădlichen Gewohnheiten und vor Unwissenheit zu schützen. "35

"Der Staat trifft die Maßnahmen zur Entwicklung der körperlichen und geistigen Gesundheit der türkischen Staatsbürger jeden Alters und fördert die Verbreitung des Sports unter den Massen.

Der Staat schützt den erfolgreichen Sportler." ${ }^{.36}$

In der Türkei wurde besonders nach den 80 er Jahren die Zahl der Sportanlagen, bzw. der Sportgerăte und anderen Ausstattungen erhöht und in Anatolien im Gleichgewicht gestreut. Diese Bauten erfolgten zum Teil in den Schulen, aber besonders in den Hochschulen.

${ }^{34}$ Siehe S. Dilik, Sosyal Yardımlaşma ve Dayanışmayı Teşvik Kanunu (Kommentar zum Gesetz zur Förderung der gegenseitigen Hilfeleistung und Solidarität), in: Çimento Işveren (Zement-Arbeitgeber), Bd. 1, Nr. 2, März 1987, S. 8-19.

35 Verfassungsgesetz, Art. 58

36 Verfassungsgesetz, Art. 59. 
In allen Fakultăten der Universităten wurde der Sportunterricht, wie es im sonsten in Grund- und hobheren Schulen der Fall ist, obligatorisch. Die Studenten und außerhalb der Hochschulen die Bevölkerungsmassen wurden andererseits zum Sporttreiben ermuntert.

Besonders nach den 90 er Jahren wurden an den Universităten viele Sporthochschulen oder -Abteilungen gegrindet, um Sportlehrer auszubilden. Erfolgreiche Sportler wurden an Sporthochschulen immatrikuliert.

Es muB aber hinsichtlich des Sports noch viel geleistet werden, um das europaische Niveau zu erreichen.

In der Türkei wird, sei es durch polizeiliche Maßnahmen, sei es durch Aufklärung und Bildung, viel angestrebt, um die Jugendlichen vor schădlichen Gewohnheiten, wie Alkoholismus, Betäubungsmitteleinnahme, Glückspiel usw. zu schützen. Trotzdem tendieren sich diese schädlichen Gewohnheiten besonders in größeren Städten zu vermehren. Hier muß also auch viel gemacht werden.

\section{Schutz der im Ausland arbeitenden türkischen Staatsbürger}

Der Staat soll nach türkischer Verfassung "die notwendigen Maßnahmen zur Gewăhrleistung der Einheit der Familie der im Ausland arbeitenden türkischen Saatsbürger, der Erziehung ihrer Kinder, ihrer kulturellen Bedürfnisse und ihrer sozialen Sicherheit, zum Schutz ihrer Bindungen an das Vaterland und zur Hilfestellung bei ihrer Rückkehr in die Heimat treffen. ${ }^{37}$

Zum Schutze der türkischen Arbeiter im Ausland wurden schon seit den 60 er Jahren mit vielen Staaten, die türkische Arbeiter beschäftigen, bilaterale Abkommen für soziale Sicherheit sowie für Arbeitsvermittlung getroffen.

Bei den türkischen Botschaften und Konsulaten errichtete man Abteilungen für Arbeiterangelegenheiten.

Die türkischen Arbeiter im Ausland werden auf verschiedene Weise auf ihre Rechte und Pflichten im Gastgeberland aufmerksam gemacht und aufgeklärt. Soweit wie der Personalzustand ermöglicht, leistet man ihnen Beratungsdienste sowie Hilfe bei der Lősung ihrer persönlichen und familiären Probleme. Man leistet ihnen auch Rechtsbeistandsdienste, falls es um wichtige Angelegenheiten handelt, die alle türkischen Arbeiter interessieren.

Zur Befriedigung der kulturellen Bedürnisse der türkischen Staatsbürger sowie zur Pflegung ihrer Bindungen an die Türkei organisiert man in Gastgeberländern viele kulturelle Veranstaltungen und Tätigkeiten.

Für die türkischen Kinder im Schulalter schickt man nach Ländern, die intensiv türkische Arbeiter beschäftigen, Lehrer für Türkisch-Unterricht. Ebenso sendet man diesen Ländern Geistliche zur Befriedigung der religiösen Bedürfnisse. In Todesfällen sorgt man

${ }^{37}$ Verfassungsgesetz, Art. 62. 
bei Bedürftigkeit und bestimmten Umständen dafür, daß die Leiche in die Türkei transportien wird.

Die Einheit der Familie der im Ausland arbeitenden türkischen Staatsbürger konnte in Zusammenarbeit mit Gastgeberländern im Rahmen der Familienzusammenführung in den ersten Perioden der Auswanderung befriedigend gewăhrt werden. Da aber die Gastgeberlănder mit der Zeit die Einwanderung stoppen wollten, bzw. dafür strenge Maßnahmen getroffen haben, konnte man die Politik der Familienzusammenführung nicht mehr erfolgreich durchsetzen.

\section{Grenze der sozialen und wirtschaftlichen Rechte}

Die türkische Verfassung hat in realistischer Haltung den sozialen und okonomischen Rechten eine Grenze gesetzt. Der Artikel, der diese Grenze setzt, lautet:

"Der Staat erfüllt seine in den sozialen und wirtschaftichen Bereichen durch die Verfassung bestimmten Aufgaben unter Beachtung des Schutzes der wirtschaftlichen Stabilităt in dem Maße, wie die Finanzquellen ausreichen." 38

Manche Autoren meinen, daß die Verfassung mit dieser Bestimmung dem Staat einen Vorwand gibh, seine Aufgaben nicht zu erfüllen. Nach unserer Meinung ist diese Begrenzung eine Notwendigkeit. Denn von heute auf morgen konnen die in der Verfassung vorgesehenen Ziele und Grundsätze nicht erreicht werden. Um so mehr gilt das für ein Land wie die Türkei, wo das Sozialprodukt, bzw. die Einnahmen des Staates sehr begrenzt sind. Man kann aber andererseits niemals sagen, daß diese Aufgaben des Staates schon verwirklicht seien und deshalb bleibe dem Staat nichts mehr zu tun ubrig. Denn je mehr sich die Quellen des Landes erhöhen, desto höher und umfassender sind die Ziele auszudeuten. Deshalb ist nach unserer Meinung die obige Bestimmung der türkischen Verfassung nur positiv zu beurteilen. Der Staat sollte aber diese Bestimmung nicht mißbrauchen, um seine sozialen Aufgaben zu versäumen.

\section{Schlußfolgerung}

Als Zusammenfassung läßt sich festhalten: Die Grundsătze der türkischen Verfassung hinsichtlich der Sozialstaatlichkeit reichen aus, um ein recht perfektes System für einen Sozialstaat zu realisieren. Die Verwirklichung dieses System erfolgte jedoch in der Tat nur teilweise. Die Sozialpolitik, die die Türkei ausführte, reiche zur Erfüllung der sozialstaatlichen Ziele der Verfassung nicht aus. Der Grund dafür liegt zweifellos zum Teil an der unzulänglichkeit der wirtschaftlichen Hilfsquellen der Türkei als ein Land, das seine Entwicklung noch nicht in vollem Maß vollbringen konnte. Man kann aber nicht behaupten, daß man dazu alles getan habe, was im Rahmen der Möglichkeit stand. Die Versäumnisse und Müßigkeit eines schlecht funktionierenden Verwaltungsapparates spielten dabei sicherlich eine große Rolle. -

${ }^{38}$ Verfassungsgesetz, Art. 65. 